\title{
The Impact of Ramucirumab Treatment on Survival and Quality of Life in Patients with Gastric Cancer
}

This article was published in the following Dove Press journal:

Cancer Management and Research

\author{
Rutika Mehta \\ Anuhya Kommalapati \\ Richard D Kim \\ Department of Gastrointestinal \\ Oncology, H. Lee Moffitt Cancer Center, \\ Tampa, FL, USA
}

\begin{abstract}
Gastric cancer is the sixth most common cancer and is known to be the fifthleading cause of cancer-related deaths globally in 2018. Systemic therapy remains the only curative option in advanced gastric carcinoma with the primary goal of improving the Health-related Quality of Life (HRQoL) (including palliation of symptoms such as dysphagia) and prolonging overall survival. Recently, ramucirumab is approved by the United States Food and Drug Administration (US-FDA) as a second-line agent either as monotherapy or in combination with paclitaxel in advanced or metastatic gastric and gastro-esophageal junction adenocarcinoma patients who have progressed on prior treatment with fluoropyrimidine or platinum containing chemotherapy. HRQoL is a subjective term that typically constitutes four components - psychological, social, occupational and physical well being. This has been evaluated as secondary endpoint in the pivotal Phase III trials with ramucirumab. HRQoL measurement can potentially provide additional information for clinical decision making beyond that of traditional medical outcomes. The present work is primarily focused on discussing HRQoL in gastric cancer patients and the impact of ramucirumab on the HRQoL in the patients with advanced gastric cancer. We also summarized the studies that evaluated the benefits of systemic therapies on HRQoL in advanced gastric cancer.
\end{abstract}

Keywords: ramucirumab, gastric cancer, gastroesophageal junction cancer, quality of life

\section{Introduction}

Gastric cancer is the sixth most common cancer and is known to be fifth-leading cause of cancer-related deaths globally in 2018. ${ }^{1}$ Systemic therapy remains the only curative option in advanced gastric carcinoma with the primary goal of improving the Healthrelated Quality of Life (HRQoL) (including palliation of symptoms such as dysphagia) and prolonging overall survival (OS). Fluoropyrimidines in combination with platinum agents remain as preferred first line therapy in non-HER2 positive gastric adenocarcinomas. ${ }^{2}$ In tumors that are highly positive for HER2 (3+ on immunohistochemical staining) or positive of florescent-in situ hybridization (FISH), a combination of chemotherapy and trastuzumab is the standard of care. ${ }^{2}$

Ramucirumab, a fully monoclonal IgG1 antibody targeting Vascular Endothelial Growth Factor Receptor 2 (VEGFR2) is approved by the United States Food and Drug Administration (US-FDA) as a second-line agent either as monotherapy or in combination with paclitaxel in advanced or metastatic gastric and gastro-esophageal junction (GEJ) adenocarcinoma patients who have progressed on prior treatment with fluoropyrimidine or platinum containing chemotherapy, based on the results of two phase III trials (REGARD, RAINBOW). In the REGARD trial, ramucirumab was compared to best supportive care alone in 355 gastric and GEJ cancer patients
Correspondence: Richard D Kim

H. Lee Moffitt Cancer Center, 12902

Magnolia Drive FOB-2, Tampa, FL

33612 , USA

Tel $+\mid$ 8/3-745-1277

Fax +1 813-449-8553

Email richard.kim@moffitt.org 
who had progressed on prior fluoropyrimidine or platinum-containing regimen. The median OS was 5.2 months as compared to 3.8 months in those in the placebo group. ${ }^{3}$ In the RAINBOW trial, the combination of ramucirumab and paclitaxel was compared against paclitaxel alone in 665 patients with gastric and GEJ cancer after progression on first line treatment. The OS was significantly longer in the combination arm (9.6 months) as compared to the paclitaxel alone arm (7.4 months $)^{4}$

However, even with improvement in OS, we are not curing most of advanced gastric cancer patients leading to more awareness about HRQoL. The present work is primarily focused on discussing HRQoL in gastric cancer patients and the impact of ramucirumab on the HRQoL in the patients with advanced gastric cancer. We also summarized the studies that evaluated the benefits of systemic therapies on HRQoL in advanced gastric cancer.

\section{HRQoL Questionnaires Used in Oncologic Practice and Gastric Cancer Clinical Trials}

HRQoL is a subjective term that typically constitutes four components - psychological, social, occupational and physical wellbeing of an individual. ${ }^{5}$ One or more of these components are essentially disturbed when an individual is diagnosed with a terminal disease such as cancer. This is particularly true in case of dismal cancers such as advanced gastric cancer. A pooled analysis by $\mathrm{Chu}$ et al demonstrated that the baseline HRQoL significantly predicted OS in advanced gastric cancer patients. ${ }^{6}$

Though several questionnaires are developed to assess the HRQoL, few have been validated in gastric cancer care. Most commonly used questionnaire in oncology patients is the European Organization for Research and Treatment of Cancer (EORTC) QLQ-C30 which incorporates patients' symptoms, functional and global health status. Another questionnaire, The Functional Assessment of Cancer TherapyGeneral (FACT-G), is commonly used which incorporates social and emotional factors ${ }^{7,8}$ Though these questionnaires have been validated in various cancers, as our experience evolved, it is obvious that some aspects of QoL questioners are to be disease-specific. For instance, in advanced gastroesophageal cancer, a prospective randomized trial that evaluated two systemic chemotherapy regimens showed differences in median OS and PFS despite lack of benefit in HRQoL (except at week 24) by using EORTC QLQ-C30 questionnaire. ${ }^{9}$ The lack of benefit in terms of HRQoL may be attributed to a lack of focus on disease-specific symptoms such odynophagia, dysphagia and cachexia-physical image.

In gastric cancer there are at least three validated gastric cancer-specific quality of life questionnaires including EORTC QLQ-STO22 (22-item supplement), FACT-Gastric cancer (FACT-Ga) and Dysfunction after Upper Gastrointestinal Surgery for Cancer (DAUGS32). Blazeby et al developed and validated a 22-item supplement (STO 22) along with the general EORTC QLQ-C30 questionnaire. ${ }^{10}$ This STO 22 supplement primarily focuses on gastrointestinal symptoms such as perception to food, gastroesophageal reflux issues, odynophagia, dysphagia, and early satiety. The supplement also includes emotional and social issues such as the patient's thoughts towards the illness and eating issues in presence of other people.

FACT-Ga questionnaire is another 19 -factor tool that was developed in conjunction with FACT-G general cancer questionnaire. $^{11,12}$ Similar to STO-22 supplement, this FACT-Ga questionnaire includes questions pertinent to abdominal pain, energy; in addition to emotional issues such as patient response to illness and body image. This FACT-Ga questionnaire has also been well-studied and validated internationally. ${ }^{13,14}$ Another 32-item questionnaire, DAUGS32 was developed by Nakamura et al to evaluate the QoL in gastric cancer patients who had surgical resection of the primary tumor. ${ }^{15}$ The questionnaire primarily comprises of symptoms pertinent to gastrointestinal system only - acid reflux, nausea, vomiting, digestion, etc. It is important to note that this questionnaire is designed and validated only in gastric cancer patients who had surgical resection and so not applicable in non-surgical gastric cancer patients. Moreover, it is validated in Japanese cohort only and so its international use is questionable. ${ }^{15}$

In short, clinicians and researchers have various options to determine the HRQoL outcome- ranging from generalized cancer questionnaires to highly-selective ones for gastric cancer. Though having multiple tools is beneficial to pick the suitable one for a particular study, comparison of outcomes of various studies that used different tools is a difficult task. Table 1 summarizes the key differences in QoL questionnaires commonly used in the management of gastric cancer.

\section{Global Utility of HRQoL in Clinical Trials Involving Gastric Cancer Patients}

For a disease such as gastric cancer that has limited OS, HRQoL is of significant importance to determine the goals 
Table I Comparison of Health-Related Quality of Life (HRQoL) Questionnaires Specific to Gastric Cancer

\begin{tabular}{|l|l|}
\hline $\begin{array}{l}\text { Name of the } \\
\text { Questionnaire }\end{array}$ & \\
\hline EORTC & - 22-items supplement developed to be used in \\
QLQ-STO22 & $\begin{array}{l}\text { conjunction with QLQ-30 } \\
\text { - } 5 \text { scales and four single item measures } \\
\text { - Primary focus: patient-symptoms } \\
\text { - Internationally validated }\end{array}$ \\
& $\begin{array}{l}\text { Used in any kind of treatment modality } \\
\text { (systemic therapy/surgery) }\end{array}$ \\
\hline FACT-Ga & $\begin{array}{l}\text { I9-factor questionnaire based on FACT-G } \\
\text { - Primary focus: physical and emotional symptoms } \\
\text { - Internationally validated } \\
\text { - Used in any kind of treatment modality (sys- } \\
\text { temic therapy/surgery) }\end{array}$ \\
\hline DAUGS32 & $\begin{array}{l}\text { - Stand-alone 32-factor questionnaire } \\
\text { - Primary focus: Gastrointestinal dysfunction in } \\
\text { post-surgical patients } \\
\text { Validated in Japanese population } \\
\text { - Used only in patients who had surgical resection } \\
\text { for gastric carcinoma }\end{array}$ \\
\hline
\end{tabular}

Abbreviations: EORTC QLQ, European Organization for Research and Treatment of Cancer- Quality of Life Questionnaire-Core 30; FACT-Ga, Functional Assessment of Cancer Therapy-General; FACT-G, Functional Assessment of Cancer TherapyGeneral; DAUGS32, Dysfunction after Upper Gastrointestinal Surgery for Cancer.

of care and appropriate treatment strategy. However, HRQoL reporting is not very robust in gastric cancer trials. In a systematic review involving the studies that reported HRQoL measures, it is interesting that only 37 studies reported HRQoL questionnaire among 164 randomized controlled trials that investigated palliative systemic therapy for gastroesophageal cancers. ${ }^{16}$ The systemic review reported the quality of reporting as an adjusted checklist score. According to the score, QoL reporting in the studies were classified as "very limited," "limited," and "probably robust." Based on this score, only a quarter of the studies were considered to be "probably robust." The EORTC QLQ-C30 was the most common tool used and reported in about $87 \%$ of the studies. The clinical significance of the HRQoL findings was reported in nearly $40 \%$ of the studies. Sadly, there was no improvement in the quality of reporting of HRQoL noted over the years.

A significant proportion of patients with gastric cancer has peritoneal metastases only and typically not considered to have measurable disease making them ineligible for a clinical trial. ${ }^{17}$ Unfortunately, these patients have recurrent need for paracentesis, bouts of bowel obstruction, poor nutrition all contributing adversely to their HRQoL. Despite the significant impact on HRQoL from the burden of peritoneal disease, randomized clinical trials that report HRQoL are likely not capturing this subset of gastric cancer patients due to their exclusion from clinical trials. This underscores the need for a better quality in reporting HRQoL in patients with gastric cancer. While endpoints such as median OS, progression free survival (PFS) and overall response rate (ORR) are very valid in larger studies for patients with metastatic gastric cancer, HRQoL is also a significant endpoint that must be accounted for and reported effectively in larger clinical trials for this patient population

Another study evaluated the role of self-reported QoL questionnaire in 254 advanced gastric carcinoma patients that were enrolled on three different clinical trials. ${ }^{18}$ Among these 254 patients, 65\% completed baseline EORTC QLQC30 questionnaire. Of the individual scores, the global QoL, physical and social functioning of patients had significant impact on median OS. Of these, social functioning remained significant even in multivariate analysis (HR:0.40, 95\% CI: $0.23-0.64, \mathrm{p}=0.001)$. The one-year survival rate of those reporting better social functioning was about $45 \%$ as compared to $\sim 18 \%$ for those that reported poor social functioning. Another pooled analysis involving three randomized, prospective trials $(n=1080)$ evaluated the role of pre-treatment QoL in predicting OS in patients with advanced gastric and gastroesophageal cancer. ${ }^{6}$ The authors have identified four independent variables that determined OS based on the multivariate analysis, which included baseline performance status, elevated alkaline phosphatase $\geq 100 \mathrm{U} / \mathrm{L}$, peritoneal, and liver metastases. The researchers demonstrated that pretreatment QoL, physical and role functioning predicted median OS in these patients (all $\mathrm{p}<0.01$ ). In addition, few other studies evaluated the role of palliative and systemic chemotherapy in improving the HRQoL in gastric and gastro-esophageal cancer. ${ }^{19-21}$

Targeted therapy trials that evaluated the benefits of bevacizumab (AVAGAST trial) and apatinib either alone or in combination with chemotherapy in gastric cancer patients also focused on HRQoL benefits as secondary endpoints. ${ }^{22,23}$ The phase III AVAGAST trial evaluated the role of combination therapy of bevacizumab and fluoropyrimidine/cisplatin doublet in metastatic gastric cancer patients. The study used the EORTC QLQ-C30 and STO22 supplement to determine the HRQoL benefits. Unfortunately, the drug regimen did not show any meaningful benefits in HRQoL at the end of study period. A Phase II trial that evaluated apatinib in chemotherapy refractory metastatic gastric cancer used EORTC QLQC30 questionnaire. ${ }^{23}$ Apatinib arm demonstrated better quality 
sleep $(\mathrm{p}=0.002)$ and a trend towards an improvement in cognitive function whereas other parameters remained the same across the study arms. ${ }^{23}$

Apart from these EORTC QLQ-C30 and FACT-Ga questionnaires, other general QoL questionnaires have been used in determining the QoL improvement in gastric cancer. Recently, a pooled analysis $(n=654)$ of a couple of randomized, prospective trials evaluating the benefits of fluoropyrimidine and cisplatin doublet used EQ-5D-3L questionnaire. ${ }^{24}$ EQ-5D-3L is an instrument developed by EuroQol group to measure health in 5- dimensions at 3 levels. ${ }^{25}$ The pooled analysis concluded that the patients who had baseline high EQ-5D-3L score (better HRQoL) had a better median OS $(p<0.01)$ and PFS $(p=0.04)$ as compared to that of counterparts on multivariate analysis. In addition, an interesting study by Rha et al, aimed at identifying the unmet needs faced by gastric cancer patients. ${ }^{26}$ The study identified that unmet needs in health information, physical and daily living domain significantly contributed to HRQoL.

While the studies that evaluated the role of systemic therapies in improving HRQoL in gastric cancer patients are limited, data have been more robust on the benefits of surgical therapies in HRQoL. ${ }^{27}$ A Canadian retrospective study that evaluated the role of surgery in gastric cancer patients showed that surgical resection contributes to compromised QoL in immediate post-operative period but returned to normal in six months. ${ }^{28}$ Similar results were seen in a prospective trial from Sloan Kettering involving 134 gastric cancer patients. ${ }^{29}$ The study used EORTC QLQC30 questionnaire and QLQ-STO22 supplement to analyze the benefits. The study showed that the HRQoL worsened in immediate post-operative period, which recovered to baseline in $65-80 \%$ of patients. Moreover, HRQoL outcome primarily depended on the type of surgical resection. For example, proximal gastrectomy resulted in inferior outcomes as compared to distal and total gastrectomy.

\section{HRQoL Studies Using Ramucirumab in Gastric Cancer}

In terms of HRQoL, HRQoL was measured at baseline and at six week mark using EORTC QLQ-C30 questionnaire in both REGARD and RAINBOW studies. ${ }^{3}$ In the REGARD study only half of the ramucirumab subset (48\%) reported the QoL data whereas data was reported by only a quarter of the placebo subset. Despite the statistically significant improvement in median OS, ramucirumab showed non-statistically significant improvement in global HRQoL as compared to that of placebo $(\mathrm{p}=0.23)$. In combination with paclitaxel (RAINBOW trial), ramucirumab did not show any worsening of HRQoL as compared to that of placebo. ${ }^{4}$ That being said, a pooled analysis of the two global randomized, prospective trials (RAINBOW and REGARD trials) explored the association of HRQoL (using QLQ-C30 questionnaire, 0-100 point scale-higher scores on functional and symptoms based questions imply poor performance while on the global health-status questions, higher score implies better performance) with overall radiological response, treatment associated adverse events, and performance status. ${ }^{30}$ This analysis clearly demonstrated that HRQoL was positively correlated with performance status of the patient, tumor response to therapy irrespective of measurable and non-measurable disease. The QLQ-C30 scales that did not change much with performance status were for nausea/ vomiting, dyspnea, insomnia, and diarrhea. Similar to performance status, HRQoL positively correlated with objective response - the patients who had complete or partial response had better HRQoL scores on ramucirumab arms and understandably, the patients with progressive disease had worsened HRQoL score.

In this pooled analysis, only a five-point change in physical functioning scores predicted a change in performance status when compared at six weeks from baseline. Most other scales including global QoL, role functioning, fatigue and pain needed $10-15$ point change to predict for change in performance status. This demonstrates that performance status does not capture all facets of QoL in gastric cancer patients. Moreover, it is interesting to see that the patients with non-measurable disease reported similar scores on the QLQ-C30 as patients with measurable disease. This leads us to think that our notion that patients with peritoneal disease only (and typically ones who have non-measurable disease) have poorer HRQoL may not be correct. Studies have shown that patients with peritoneal metastasis have shorter median OS as compared to patients without peritoneal metastasis and have higher risk for mortality. ${ }^{17}$ It might be possible that patients with peritoneal metastasis might have had improvement in their symptoms from prior systemic treatment. Moreover, given that only patients with ECOG 0 or 1 and that have normal laboratory values are permitted on the study, there might have been a selection bias for patients that had controlled symptoms in spite of peritoneal metastases. Interestingly, there was improvement in emotional functioning scores in patients with non-measurable disease who had progressive disease. 
When the treatment-associated adverse events were compared to QLQ-C30 scores from baseline to six weeks, it was noted that investigator reported neuropathy was significantly associated with worsening scores across several scales including fatigue, nausea, vomiting, appetite loss, physical-, role-, social- and emotional-functioning. Neuropathy as a treatmentassociated adverse event was reported in the RAINBOW trial. Any grade neuropathy was noted in $40 \%$ of patients and grade III neuropathy was reported in $6 \%$ of patients being treated on the RAINBOW trial. ${ }^{4}$ The patients with low appetite were found to have proportional low scores on emotional functioning, fatigue, and appetite loss. Also, the patient reported symptoms on the QLQ-C30 were associated with investigator reported symptoms such as loss of appetite, nausea/vomiting, fatigue and diarrhea. Thus, the QLQ-C30 tool is a fair reflection of treatment-associated adverse events in gastric cancer.

Recently, HRQoL was also evaluated in another randomized phase III trial (RAINFALL trial) that evaluated the combination of ramucirumab and cisplatin/fluoropyrimidine doublet in the first-line setting in advanced gastric cancer. ${ }^{31,32}$ In this trial the ramucirumab arm did not result in a significant prolongation in the overall survival as compared to the placebo (11.2 months versus 10.7 months; hazard ratio $0.962 ; p=0.74)$ even though there was PFS benefit. But the trial used EORTC QLQ-C30 tool to evaluate the QoL, which showed that time to sustained deterioration in QoL was similar in ramucirumab and placebo group. In addition, the rate of improved or stable changes in the different scales for QoL was similar across both arms. ${ }^{31}$

In due course of time, we will have information on the impact of QoL by other agents such as Trifluridine/tipiracil (FTD/TPI) and apatinib which have been studied in this space. The TAGS study has not yet published the HRQoL data. ${ }^{33}$ The data from ANGEL was recently released to have not resulted in OS advantage in the entire population, although some benefit was noted when apatinib (now also known as rivoceranib) was used in 4th line or beyond setting. ${ }^{34}$ The ATTRACTION-2 trial of nivolumab did not collect HRQoL data. ${ }^{35}$ Although data on HRQoL studies in gastric cancer patients is limited, available data indicate that the course of the disease and the treatments of gastric cancer may show impact on patients' HRQoL. Lack of information on HRQoL may be attributed to reporting bias where the researchers may have chosen not to disclose the HRQoL outcomes due to lack of benefit. Another possible explanation is lack of universal questionnaire and the percentage change in score from baseline causes difficulty in evaluating the HRQoL outcomes in global randomized, prospective trials. Hence, having a generalized or universal HRQoL questionnaire could help us in comparing the benefits of various therapies in a better way. However, in multinational trials, principal investigators may be well-versed with their local QoL questionnaires - developing and validating a new, novel, and universal HRQoL questionnaire pertinent to gastric cancer comes with challenges.

\section{Conclusion}

Angiogenesis plays a key role in the tumorigenesis of gastric cancer. Ramucirumab has shown OS benefit either as monotherapy or in combination with paclitaxel as second line treatment for gastric cancer patients. Practical understanding of gastric cancer patients demonstrated that HRQoL outcome is one of the key factors that still remained as an unmet need. Although EORTC QLQ-C30 and FACT-G scores are generic scales and not designed specifically for gastric and gastroesophageal cancer patients, studies show that they provide a fair estimation of the HRQoL in spite of special symptoms of interest in this patient population. These HRQoL questionnaires and DAUGS32 tool have been extensively utilized in studies that evaluated the role of surgical therapies in gastric cancer patients. On the contrary, studies determining the benefit of systemic and targeted therapies on HRQoL in gastric cancer patients have been limited. The quality of reporting of HRQoL in gastric cancer studies is not always robust, which underscores the need for improvement at this end. Future studies should focus on developing a universal HRQoL tool to maintain the uniformity across the studies, which makes QoL a key clinical outcome measure in gastric cancer clinical trials. There have been several initiatives across the world to develop consensus guidelines for recommendations of items to be included in clinical trials incorporating patient reported outcomes. A Delphi study resulted in the COSMIN (COnsensus-based Standards for the selection of health status Measurement INstruments) checklist that would be useful to define measurements for health outcomes. ${ }^{36}$ The Standard Protocol Items: Recommendations for Interventional Trials Patient-Reported Outcome (SPIRIT-PRO) is another global consensus guideline that provides recommendations on items to be included in clinical trial protocols that incorporate patient reported outcomes as an endpoint. ${ }^{37}$ The goal of these guidelines is to ensure standardization of tools being used across studies and compare the findings.

\section{Disclosure}

Dr. Richard D Kim received honorarium from Lilly, BMS and Bayer, outside of the submitted work. Dr. Rutika 
Mehta received honorarium from Lilly, during the conduct of the study; received travel reimbursement from BMS. She is part of the Advisory Board for Taiho Oncology and offers consultancy to Alphasights, outside of the submitted work. The authors report no other conflicts of interest for this work.

\section{References}

1. Bray F, Ferlay J, Soerjomataram I, Siegel RL, Torre LA, Jemal A. Global cancer statistics 2018: GLOBOCAN estimates of incidence and mortality worldwide for 36 cancers in 185 countries. CA Cancer J Clin. 2018;68(6):394-424. doi:10.3322/caac.21492

2. Salati M, Orsi G, Smyth E, et al. Gastric cancer: translating novels concepts into clinical practice. Cancer Treat Rev. 2019;79:101889. doi:10.1016/j.ctrv.2019.101889

3. Fuchs CS, Tomasek J, Yong CJ, et al. Ramucirumab monotherapy for previously treated advanced gastric or gastro-oesophageal junction adenocarcinoma (REGARD): an international, randomised, multicentre, placebo-controlled, Phase 3 trial. Lancet. 2014;383(9911):31-39. doi:10.1016/S0140-6736(13)61719-5

4. Wilke H, Muro K, Van Cutsem E, et al. Ramucirumab plus paclitaxel versus placebo plus paclitaxel in patients with previously treated advanced gastric or gastro-oesophageal junction adenocarcinoma (RAINBOW): a double-blind, randomised phase 3 trial. Lancet Oncol. 2014;15(11):1224-1235. doi:10.1016/S1470-2045(14)70420-6

5. Fallowfield L. Quality of life: a new perspective for cancer patients. Nat Rev Cancer. 2002;2(11):873-879. doi:10.1038/nrc930

6. Chau I, Norman AR, Cunningham D, Waters JS, Oates J, Ross PJ. Multivariate prognostic factor analysis in locally advanced and metastatic esophago-gastric cancer-pooled analysis from three multicenter, randomized, controlled trials using individual patient data. J Clin Oncol. 2004;22(12):2395-2403. doi:10.1200/JCO.2004.08.154

7. Aaronson NK, Ahmedzai S, Bergman B, et al. The European Organization for Research and Treatment of Cancer QLQ-C30: a quality-of-life instrument for use in international clinical trials in oncology. J Natl Cancer Inst. 1993;85(5):365-376. doi:10.1093/ jnci/85.5.365

8. Cella DF, Tulsky DS, Gray G, et al. The Functional Assessment of Cancer Therapy scale: development and validation of the general measure. J Clin Oncol. 1993;11(3):570-579. doi:10.1200/JCO.1993.11.3.570

9. Webb A, Cunningham D, Scarffe JH, et al. Randomized trial comparing epirubicin, cisplatin, and fluorouracil versus fluorouracil, doxorubicin, and methotrexate in advanced esophagogastric cancer. J Clin Oncol. 1997;15(1):261-267. doi:10.1200/JCO.1997.15.1.261

10. Blazeby JM, Conroy T, Bottomley A, et al. Clinical and psychometric validation of a questionnaire module, the EORTC QLQ-STO 22, to assess quality of life in patients with gastric cancer. Eur J Cancer. 2004;40(15):2260-2268. doi:10.1016/j.ejca.2004.05.023

11. Garland SN, Pelletier G, Lawe A, et al. Prospective evaluation of the reliability, validity, and minimally important difference of the functional assessment of cancer therapy-gastric (FACT-Ga) quality-of-life instrument. Cancer. 2011;117(6):1302-1312. doi:10.1002/cncr.25556

12. Eremenco SL, Cashy J, Webster K, et al. FACT-Gastric: A new international measure of QOL in gastric cancer. J Clin Oncol. 2004;22(14_suppl):8123. doi:10.1200/jco.2004.22.90140.8123

13. Zhou HJ, So JB, Yong WP, et al. Validation of the functional assessment of cancer therapy-gastric module for the Chinese population. Health Qual Life Outcomes. 2012;10:145. doi:10.1186/1477-7525-10-145

14. Debb SM, Arnold B, Perez B, Cella D. Validation of the FACT-Gastric cancer quality of life questionnaire for use in Spanish-speaking countries. Psycho-Oncology. 2011;20(1):19-27. doi:10.1002/pon.v20.1
15. Nakamura M, Hosoya Y, Yano M, et al. Extent of gastric resection impacts patient quality of life: the Dysfunction After Upper Gastrointestinal Surgery for Cancer (DAUGS32) scoring system. Ann Surg Oncol. 2011;18(2):314-320. doi:10.1245/s10434-010-1290-y

16. Ter Veer E, van Kleef JJ, Sprangers MAG, Haj Mohammad N, van Oijen MGH, van Laarhoven HWM. Reporting of health-related quality of life in randomized controlled trials involving palliative systemic therapy for esophagogastric cancer: a systematic review. Gastric Cancer. 2018;21(2):183-195. doi:10.1007/s10120-018-0792-3

17. Wei J, Wu N-D, Liu B-R. Regional but fatal: intraperitoneal metastasis in gastric cancer. World $j$ Gastroenterol. 2016;22(33):7478-7485. doi:10.3748/wjg.v22.i33.7478

18. Park SH, Cho MS, Kim YS, et al. Self-reported health-related quality of life predicts survival for patients with advanced gastric cancer treated with first-line chemotherapy. Qual Life Res. 2008;17 (2):207-214. doi:10.1007/s11136-008-9307-8

19. van Kleef JJ, Ter Veer E, van den Boorn HG, et al. Quality of life during palliative systemic therapy for oesophagogastric cancer: systematic review and meta-analysis. J Natl Cancer Inst. 2019. doi:10.1093/jnci/djz133

20. Wagner AD, Syn NL, Moehler M, et al. Chemotherapy for advanced gastric cancer. Cochrane Database Syst Rev. 2017;8:Cd004064.

21. Al-Batran SE, Ajani JA. Impact of chemotherapy on quality of life in patients with metastatic esophagogastric cancer. Cancer. 2010;116 (11):2511-2518. doi:10.1002/cncr.25064

22. Ohtsu A, Shah MA, Van Cutsem E, et al. Bevacizumab in combination with chemotherapy as first-line therapy in advanced gastric cancer: a randomized, double-blind, placebo-controlled phase III study. J Clin Oncol. 2011;29(30):3968-3976. doi:10.1200/JCO. 2011.36.2236

23. Li J, Qin S, Xu J, et al. Apatinib for chemotherapy-refractory advanced metastatic gastric cancer: results from a randomized, placebo-controlled, parallel-arm, phase II trial. J Clin Oncol. 2013;31(26):3219-3225. doi:10.1200/JCO.2013.48.8585

24. Abdel-Rahman O. Prognostic impact of baseline quality of life status among patients with advanced gastric cancer; results from two randomized studies. Expert Rev Pharmacoecon Outcomes Res. 2019;1-5. doi:10.1080/14737167.2019.1596027

25. Group TE. EuroQol-a new facility for the measurement of healthrelated quality of life. Health Policy. 1990;16(3):199-208. doi:10.1016/0168-8510(90)90421-9

26. Rha SY, Lee HJ, Lee J. Unmet needs in the physical and daily living domain mediates the influence of symptom experience on the quality of life of gastric cancer patients. Support Care Cancer. 2019. doi:10.1007/s00520-019-04954-3

27. McCall MD, Graham PJ, Bathe OF. Quality of life: a critical outcome for all surgical treatments of gastric cancer. World J Gastroenterol. 2016;22(3):1101-1113. doi:10.3748/wjg.v22.i3.1101

28. Munene G, Francis W, Garland SN, Pelletier G, Mack LA, Bathe OF. The quality of life trajectory of resected gastric cancer. J Surg Oncol. 2012;105(4):337-341. doi:10.1002/jso.22139

29. Karanicolas PJ, Graham D, Gonen M, Strong VE, Brennan MF, Coit DG. Quality of life after gastrectomy for adenocarcinoma: a prospective cohort study. Ann Surg. 2013;257(6):1039-1046. doi:10.1097/SLA.0b013e31828c4a19

30. Chau I, Fuchs CS, Ohtsu A, et al. Association of quality of life with disease characteristics and treatment outcomes in patients with advanced gastric cancer: exploratory analysis of RAINBOW and REGARD phase III trials. Eur J Cancer. 2019;107:115-123. doi:10.1016/j.ejca.2018.11.013

31. Chau I, Al-Batran S-E, Luft A, et al. 616PDQuality-of-life (QoL) results from RAINFALL: a randomized, double-blind, placebo (PL)-controlled phase III study of cisplatin (Cis) plus capecitabine (Cape) or 5FU with or without ramucirumab (RAM) as first-line therapy for metastatic gastric or gastroesophageal junction (G-GEJ) cancer. Ann Oncol. 2018;29(suppl_8). doi:10.1093/annonc/mdx807 
32. Fuchs CS, Shitara K, Di Bartolomeo M, et al. Ramucirumab with cisplatin and fluoropyrimidine as first-line therapy in patients with metastatic gastric or junctional adenocarcinoma (RAINFALL): a double-blind, randomised, placebo-controlled, phase 3 trial. Lancet Oncol. 2019;20(3):420-435. doi:10.1016/S1470-2045(18)30791-5

33. Shitara K, Doi T, Dvorkin M, et al. Trifluridine/tipiracil versus placebo in patients with heavily pretreated metastatic gastric cancer (TAGS): a randomised, double-blind, placebo controlled, phase 3 trial. Lancet Oncol. 2018;19(11):1437-1448. doi:10.1016/S14702045(18)30739-3

34. Kang Y-K, Kang WK, Di Bartolomeo M, et al. Randomized phase III ANGEL study of rivoceranib (apatinib) + best supportive case (BSC) vs placebo $+\mathrm{BSC}$ in patients with advanced/metastatic gastric cancer who failed $\geq 2$ prior chemotherapy regimens. Ann Oncol. 2019;30 (suppl_5):mdz394-034.
35. Kang Y-K, Boku N, Satoh T, et al. Nivolumab in patients with advanced gastric or gastro-oesophageal junction cancer refractory to, or intolerant of, at least two previous chemotherapy regimens (ONO-4538-12, ATTRACTION-2): a randomised, double-blind, placebo-controlled, phase 3 trial. Lancet. 2017;390(10111):246102471. doi:10.1016/ S0140-6736(17)31827-5

36. Mokkink LB, Terwee CB, Patrick DL, et al. The COSMIN checklist for assessing the methodological quality of studies on measurement properties of health status measurement instruments: an international Delphi study. Qual Life Res. 2010;19(4):539-549. doi:10.1007/ s11136-010-9606-8

37. Calvert M, Kyte D, Mercieca-Bebber R, et al. Guidelines for inclusion of patient-reported outcomes in clinical trial protocols: the SPIRIT-PRO extension. JAMA. 2018;319(5):483-494. doi:10.1001/ jama.2017.21903

\section{Publish your work in this journal}

Cancer Management and Research is an international, peer-reviewed open access journal focusing on cancer research and the optimal use of preventative and integrated treatment interventions to achieve improved outcomes, enhanced survival and quality of life for the cancer patient.
The manuscript management system is completely online and includes a very quick and fair peer-review system, which is all easy to use. Visit http://www.dovepress.com/testimonials.php to read real quotes from published authors. 